\title{
Culture of Communication Workgroup Report
}

Barbara DeFelice, Susan Haigh, Barrett Matthews, Dan Morgan, Eric L. Olson, Leslie J. Reynolds, Rachael G. Samberg, Jason Steinhauer, Mary Yess

\section{Abstract / OSI2017 Workgroup Question}

Following a common thread from throughout OSI2016, this workgroup will develop partnership proposals for this community to work together to improve the culture of communication inside academia, particularly inside research. As part of this effort, it may be important to clarify messaging surrounding the benefits and impacts of open access (OA) inside academia, particularly inside research. It may also be important to determine what resources and information are needed before this messaging can be effective, including showing the benefits of OA to a skeptical research community; addressing the many concerns of stakeholders; clearly explaining its pros and cons; and demonstrating the case for why the transition to OA is worth the trouble.

\section{Overview and Summary of}

\section{Proposal}

Fifteen years following the Berlin Open Access Initiative, the academic publishing community continues to encounter challenges in describing and discussing what is open access (OA), and what benefits and impacts it carries. The messages conveyed between and among stakeholders vary widely, and often conflict. To some librarians, for instance, OA connotes a funding model-contingent upon replacing subscriptions with article processing charges or memberships. To others, OA may be perceived as a means of conserving collections budgets by eliminating subscriptions. To certain academic authors, OA might carry the perception of favoring STEM disciplines for which more OA journals (and thus OA publishing opportunities) currently exist. For some academic publishers, OA may appear to threaten the scholarly publishing's prestige in the face of journal proliferation.

These examples are oversimplified characterizations of stakeholder opinions. Yet they underscore the important notion that we have not yet determined how to communicate effectively about OA. Indeed, these equivocal and complex conceptions of OA have yielded a culture of communication in which scholarly publishing stakeholders effectively speak different languages when trying to discuss their needs and concerns. This has resulted in tension, misunderstanding, interdisciplinary differences in experiences, and a maintenance of status quo. More than two decades into the OA movement, universal $\mathrm{OA}$ is far from realized and the current reality of OA has not lived up to our vision.

C 2017 OSI2017 Culture of Communication Workgroup. This open access article is distributed under the Creative Commons Attribution 4.0 International License. This document reflects the combined input of the authors listed here (in alphabetical order by last name) as well as contributions from other OSI2017 delegates. The findings and recommendations expressed herein do not necessarily reflect the opinions of the individual authors listed here, nor their agencies, trustees, officers, or staff. 
By simplifying how we communicate about OA, its benefits and impacts become easier to evaluate and discuss. In its simplest form, open access is an outcome: scholarship may be accessed online without cost or other barriers to access and reuse by readers. As such, OA is separate from and unencumbered by myriad pathways that could be invoked to achieve OA as well as the resulting landscape of $\mathrm{OA}$ journals. By paring down how we describe and understand $\mathrm{OA}$ in this fashion, we can more easily identify and address where stakeholder needs diverge and overlap, and collaboratively forge tangibile pathways forward. To make progress towards OA, therefore, we must address how we have come to describe and discuss $\mathrm{OA}$ itself.

This OSI workgroup, the "Culture of Communication" (CoC), was tasked with addressing this very problem. Following OSI 2016's common thread about conflicting messaging around $\mathrm{OA}$, this workgroup was asked to develop partnership proposals for this community to work together to improve the culture of communication inside academia. As discussed more fully below, we set out to accomplish these aims by articulating the needs to:

- Clarify the message about OA. Identify what $\mathrm{OA}$ is, and what it is not. OA is simply an outcome-the scholarship is freely accessible online, and freely reusable. OA is not a funding model, a peer review system, or a resulting landscape of journals.

- Create the message for communities. Simplify the message to its core values and the societal effects it enables. Specifically, open access: pro- motes innovation and progress; benefits the public good by promoting social justice and democratization, and; supports professional impact for all academic publishing stakeholders by reducing barriers to accessing scholarship.

- Communicate a simpler message. One vehicle to facilitate communication of simpler, tailored messages is storytelling. Stories are ways to communicate about OA impact and incentives, and are particularly effective as they harness the speaker's own language in doing so.

Partnerships are essential to facilitate these three changes in the culture of communication. Partners can help create the resources, guidance, and tools for stakeholders to clarify and tailor their own messages and streamline their OA storytelling. Partnerships can also promote national rewards and incentives to celebrate $\mathrm{OA}$ successes. The $\mathrm{CoC}$ working group offers recommendations below about the types of partnerships to be formed, and the specific work product and rewards these partners can create.

\section{Need for Improvement}

The CoC working group identified numerous examples of misunderstanding and miscommunication regarding $\mathrm{OA}$ from our varied experiences. Although much has changed in the past fifteen years, the misunderstandings that remain prohibit progress and limit the potential for wider ranging collaborations. The culture of communication is not so different from 2012, when Peter Suber noted: 
"My honest belief from experience in the trenches is that the largest obstacle to OA is misunderstanding." (Peter Suber, Open Access, MIT Press 2012)

"Nobody is surprised when cultural inertia slows the adoption of radical ideas. But cultural inertia slowed the adoption of OA by leading many people to mistake it for a more radical idea than it actually is."

(Peter Suber, Open Access, MIT Press 2012)

The culture that has developed is marked with:

1. Tension

2. Misunderstanding

3. Inter-disciplinary differences in experiences

4. Maintenance of business models that no longer work well

5. A reality that does not match the ideal

Many stakeholders are involved in the global shift to a more open dissemination of knowledge, and communication challenges are intrinsic given the diversity of interests. However, many of the stakeholders encounter misunderstandings within their own institutions, across different disciplines and from those with different statuses in the research and scholarly enterprise. We must develop better ways to communicate across all these stakeholders, and develop a range of tools for those who speak from vastly different backgrounds and with different concerns. To improve the current culture of communication, we need to hear a wider range of stories and to also give a wider range of stakeholders the knowledge and authority to speak for the changes they want to see.

\section{Addressing the Message}

\section{Clarifying the Message}

Ultimately, OA is simply an outcome for a scholarly object (article, book, etc.) whereby it is freely accessible online, and freely reusable. Always using this simple clarification as the basis of your message enables you to create the most appropriate message for your audience.

While OA may be aligned (or not) with the following notions, it should not be defined or explained using any of them: a specific business model, peer review criterion, cost-cutting strategy, or a resulting landscape of journals.

\section{Creating the Message}

Using the above clarification as the basis of your message enables you to more effectively create communications targeted for particular communities. For example, if your message requires you to try and enable more OA publishing at your institution, you can make it clear that you, e.g., never intend that an author must write a different type of book, or a lower-quality journal article. It is the same book and the same article because OA is simply an outcome that enables free access and reuse.

Simplifying the message first not only helps address any preconceived notions that may prevent appreciation of it, but enables you to effectively add the more complex specific and contextual information that your audience is expecting. For example, if your aim is to promote innovation and progress with your message about $\mathrm{OA}$, you can clear up any doubts that people sacrifice quality or attribution by enabling reuse and easier building on the work of others. If your 
aim is to promote social justice and democratization, then you can prevent any concerns that the content that is democratized is of any lower quality.

\section{Communicating the Message}

While the creation of messaging is a challenge, an equal challenge is how to communicate it. How do we communicate the essence and benefits of OA? What are the ways we can do so? There are a few overarching considerations.

Communicating the message must:

- Engage in a fresh way those we ask to do the communicating

- Reinforce that it is in everyone's selfinterest to push for OA

- Make the case for why this is all worth the trouble

- Provide easy, practical tools to publish OA

- Move easily along a continuum of nano- to macro-engagement (engage with individuals, engage with groups)

Who should be communicating? Everyone-all stakeholders should communicate and become OA ambassadors: authors, librarians, provosts, communication offices, professional societies, funders, and publishers. Different stages in the OA publishing process and audiences for the message require different communicators and vehicles.

Should we trust researchers to tell their own OA stories? Absolutely, we should trust the entire community, individuals and groups. But we must provide tools that easily build clear and consistent OA messaging into everyone's DNA. Responding to OA should be like respond- ing to ORCID: "Oh, that's interesting and highly beneficial; what do I need to do to take advantage of this?”

What are the vehicles we all can use? One effective vehicle is storytelling. The stories do not need to be grand, they simply must convey what resonated with the storyteller and tell the story in his/her own words. There are many benefits of using stories: they can ease communicating with the carrot of "narrative" rather than the stick of "mandates."

Scale is important to keep in mind when telling stories: one should move fluidly from the small and the personal to examples of greater impacts on the scholarly community. Advisors can lead by example and encourage their students to follow. In telling the story, sometimes it's necessary to deflect the premise ("burying the lede") - focus on the human side of the story rather than a message of "OA must happen."

Being an advocate for $\mathrm{OA}$, and publishing OA, has additional benefits - it can lead journalists to write about the storyteller. Storytelling can help encourage community building by sharing stories. It trusts and empowers communicators, enables ambassadors.

There are many good resources for examples of storytelling and actual stories. These include:

- Impact Stories

- Your Story Matters

- Open Access Success: Be Inspired by over Thirty Compelling Stories

- Global Reach: Open Access Stories $\underline{\text { Available }}$

- Open Access Success Stories 
- Open Access Success Stories Blog

- 100 Stories: The Impact of Open Access; see also Open Access: 100 Stories of Impact

- Telling Open Access Stories

Workshops are practical tools to provide top-down education and support to individuals. They can take a variety of formats and themes. An example is the University of California-Berkeley's "BRII and Brie" event. Here the library recognizes open access publications funded by the Berkeley Research Impact Initiative (BRII). The February 2017 event included remarks from the university librarian and BRIIfunded faculty members about their scholarship, and the impact of BRII and open access; a lightning round of publication introductions by attending BRII recipients in attendance; and a display and discussion of BRII-funded work.

Other examples of workshop topics include increasing impact as a researcher, how to do peer review, how to publish your dissertation, benefits of an ORCID number-all of which can carry "background" messages about OA and give options for being a good "OA citizen." Here are some examples of what others are doing and some resources to get started in creating a workshop:

- Open Access \& Scholarly Communications@, UC San Diego: Open Access Workshop Resources

- Open Access Directory's Conferences and Workshops Related to Open Access

- Electronic Information for Libraries Open Access Programme

- Frontiers Data Services Workshop in an Open Access World

- OpenAIRE Workshops
Community-based social marketing revolves around the idea that sustainable change in the behavior of members of a community has the most effect when it involves direct contact with people and is carried out within communities (moving from nano-engagement to macroengagement). Planting seeds with individuals can help to socialize the message within a community. Social-based marketing is another complex field and there are many resources to help gain an understanding as well as to start putting together a plan. While most of what has been written revolves around environmental issues, the articles can be used to extrapolate from and create a plan on socializing pro-open access behavior. Some good starting points are:

- Your Quick Guide to CommunityBased Social Marketing

- What is Community-Based Social Marketing? (And What It Means to Me and You)

Open Access Week is also an opportunity to engage on all levels and to partner with scholarly societies and open access organizations.

\section{Recommended Partnerships to Effectuate Change}

\section{Moving Forward}

The CoC workgroup parallels the direction and intentions of the OSI mission. A diverse group of stakeholders met around the same table and topic with two broad goals: analyze the well known "wicked problem" of communicating the essence and benefits of open and recommend a path forward that extends analysis into action. 
OSI and scholarly communication are, more broadly, community efforts, so almost any new developments or progress in these spaces are somewhat dialectic in nature. Communication throughout the various "camps" is essential, but this has, somewhat ironically, been a challenge within and between many of the layers of the scholarly communication process.

Our recommendation for taking the first steps toward a resolution is twofold:

1. Provide resources that can help users better understand, anticipate, and respond to the scholarly communication needs of their community; and

2. Use high-profile partnerships to institutionalize certain communication and visibility elements within scholarly communication.

\section{Help You Tell Your Story}

Throughout this report, and fully agreed upon in our workgroup, is the need for clarity when communicating about $\mathrm{OA}$, as well as several broad strategies that can help make these communications successful. Obviously, this is not enough to be the foundation of a robust communication strategy. We propose that a centralized "hub" of resources be developed as a collaborative exercise, which would feature elements beyond simply the specifics of messaging surrounding issues of scholarly communication.

We describe strategies for "scholarly storytelling", but there are resources required to implement these strategies. This hub will contain ready-made and adaptable tools for these activities, such as registering for an ORCID iD or increasing impact. It will also contain guides that help users integrate discussions and recom- mendations about open into presentations or web guides. At the start this hub can be populated with limited resources, but the various stakeholders that utilize it can add their adapted or unique elements. With appropriate curation, this can be an easily discoverable, searchable index of tools by and for a variety of users.

\section{Mapping the Culture of Your Institution} Even with the abundance of available resources, the task of communicating and contextualizing $\mathrm{OA}$ is not without complication. As we describe in the "Addressing the Message" section, most messages are not intended for, and will not succeed with, all audiences. A researcher will not respond to information or appeals about OA the same way that a dean will, and researchers in Biology may respond differently than their counterparts in English. Crafting messages for your community involves first determining who you need to communicate with and which strategies are more likely to result in behavior change for that audience.

We have already discussed some of the ways that this can be achieved, such as finding the best person in each department to serve as a node in the open conversation. But how do you get to these steps? How can you determine how the various cultures within your institution interact with each other and with the concepts of open? There is no easy answer to these questions, but we propose the development of a new tool that rests between the quickest and ideal solutions.

There are some elements of scholarly communication that can fall back on a "checklist" tactic. When dealing with people and communities, this is not likely to 
succeed. There is not one set of practices that apply to all communities or even all communities within a specific area of interest or practice. They are all different, and their relationship to the culture around them varies. Given the complicated nature of determining these interactions and forming a communication plan based on them, the ideal solution would be to collaborate with someone who is an expert in ethnography or organizational communication. They would know how to draw out and evaluate these relationships in a detailed, responsible fashion, but such a collaboration is unlikely given the already stretched resources that scholarly communicators often have at their disposal.

One solution would be an ethnographic or interviewing tool that would give users guidance on how to engage their community and draw out the information that they would need to develop a communication plan. Like the resource hub, the tool can augment its effectiveness through use and evaluation. As it is employed in various contexts, successes and adaptations can become part of the tool.

As far as our group knows, there is not a tool like this being used currently, though there have been limited ethnographic approaches to visualizing scholarly communication environments. We recommend at least exploring the viability of such a tool, and perhaps soliciting communities who would be willing to pilot test the method.

\section{Institutionalized Collaboration}

OSI 2017 re-implements the model that was utilized for OSI 2016, but with an additional objective: propose partnerships that connect the vital strands within the scholarly communication landscape. Our workgroup conceived several ways that institutions within scholarly communication can work together improve the culture of communication around OA.

\section{OSI as Fulcrum Event}

OSI has undertaken the responsibility to bring representatives of all stakeholders in the scholarly communication community together for the annual meeting and online forums. While there is an impressive diversity of organizations and nations included, there is a need to include more authors and researchers. This responsibility can also be an opportunity for partnership. Some cross-discipline academic conferences now partner with smaller, discipline-specific meetings that help to bring attention and attendance to both that they may not be able to obtain separately. OSI could reach out to research communities to propose synchronous meetings that could provide increased researcher participation in the meeting.

\section{OSI as Partnership Catalyst}

OSI's interstitial position can make it an ideal partnership catalyst with scholarly communication. As identified by several workgroups in OSI 2016 and OSI 2017, one of the challenges of communicating between the "silos" of scholarly communication is that the "producers"researchers are unfamiliar with the cultures of "providers," such as publishers, and vice versa. A fellowship program that facilitates an exchange of individuals between these silos could provide valuable insight and experience to begin bridging these cultural gaps.

An "Open Access Nobel Prize"

Visibility and recognition is vital to behavior change, and scholarly communication is a prime example. Recognition is focused most strongly on paywalled, premier jour- 
nals, while there has been a lack of incentive to publish in open access environments. Such an award would provide this incentive, and scholarly communication institutions like funders, publishers, com- munities, and more could collaborate to create and maintain it.

\section{Culture of Communication Workgroup:}

Barbara DeFelice, Program Director for Scholarly Communication, Dartmouth College Libraries

Susan Haigh, Executive Director, Canadian Association of Research Libraries

Barrett Matthews, State Authorizations Coordinator, The George Washington University

Dan Morgan, Digital Science Publisher, University of California Press

Eric L. Olson, Community Engagement \& Support Specialist, ORCID

Leslie J. Reynolds, Senior Associate Dean, University of Colorado Libraries

Rachael G. Samberg, Scholarly Communication Officer, UC Berkeley Library

Jason Steinhauer, Director, Lepage Center for History in the Public Interest, Villanova

University

Mary Yess, Deputy Executive Director, The Electrochemical Society

\section{End Notes}

1. Lanclos, Donna. "Ethnographic Approaches to the Practices of Scholarly Communication: Tackling the Mess of Academia." Insights 29, no. 3 (November 4, 2016). doi: http://doi.org/10.1629/uksg.316. 